\title{
Review of: "Ecology and genomic background shape the probability of parallel adaptation to climate"
}

\author{
Magdalena Bohutínská \\ 1 Charles University Prague
}

Potential competing interests: The author(s) declared that no potential competing interests exist.

Dear authors,

I read your manuscript with a great interest and I have some questions.

- Did you have any particular reason why you selected such a big windows? What is the level of nucleotide diversity/LD decay in Tinema? In my model species (average decay of genotype correlations $\sim 1 \mathrm{~kb}$, pi 0.02) such windows would be so large that the signal of environmental association would dilute and changes would be more likely due to the factors not directly related to adaptation to environment (i.e. recombination rate variation).

- Following this, have you tested for the relationship between shared genetic distance and proportion of convergent windows showing both climate-associated SNP and elevation-dependent allele-frequency changes (as identified in the chapter Climate-associated regions exhibit...)? I consider windows showing both signals as much better candidates for loci of adaptation and it would be cool to see if the pattern from Fig. 5 holds for them.

- Do you have any estimates about what fraction of convergent windows is selected due to the novel variants (as compared to standing variants)? These may better represent the 'constraint' hypothesis which you propose in introduction (I. 95)

Thank you for this inspiring work and lot of luck with the review process! 